\title{
Review on Leishmaniasis
}

\author{
Chiragkumar J Gohil ${ }^{* 1}$ and Jimishaben D Kher ${ }^{2}$ \\ ${ }^{1}$ Department of pharmaceutical chemistry, Shree Laxminarayan Dev College of Pharmacy, Bharuch, Gujarat, India \\ ${ }^{2}$ Department of Pharmacology, Shree Dhanvantary Pharmacy College, Surat, Gujarat, India
}

Received: October 16, 2017; Published: October 24, 2017

*Corresponding author: Chiragkumar J Gohil, Department of Pharmaceutical Chemistry, Shree Laxminarayan Dev College of Pharmacy, Bholav, Bharuch, India, Email: gohil2711@gmail.com

\begin{abstract}
Leishmanios is, is a disease caused by protozoan parasites of the genus Leishmania and spread by the bite of certain types of sand flies. It is a represent a complex of diseases with identical clinical and epidemiological diversity. They depending on clinical symptoms having 3 different species: cutaneous Leishmaniasis, visceral leishmaniasis, mucocutaneous leishmaniasis. The diff stage involvement to produce infection. This infection diagnosis is the depending on the severity of the diease. The cutaneous leishmaniasis is a not more dangerous compare two other types of leishmaniasis. The diff techniques are available for diagnosis purpose. The efficacy of treatment varies with the type of infecting species and resistance pattern. The persistent lack of vaccine against human leishmaniasis is a result of the poor investment in this neglected parasitosis.
\end{abstract}

Keywords : Leishmaniasis; Parasite; Cutaneous Leishmaniasis; Visceral leishmaniasis; Muco cutaneous leishmaniasis

\section{Introduction}

Leishmaniasis is a leave alone vector-borne tropical infection that is considered to be a disease of penury. Mostly concentrated in poor countries within South East Asia, East Africa and Latin America, leishmaniasis is endemic in several Mediterranean countries making this parasitosis disease for local inhabitants as well as for travellers. The protean manifestations of leishmaniasis, if convied, range from cutaneous, which if left untreat may result in disfiguring scars associated with social stigma, to likely lethal disseminated infections. Among all parasitic diseases, mortality from leishmaniasis is second to malaria, and in terms of disability adjusted life years (DALYs), the third common origin of morbidity after malaria and schistosomiasis, with children $<15$ years suffering all of the disease burden.1 The expand in the number of immunosuppressed separate, secondary to HIV infection, post transplant and chemotherapeutic agents and the recently introduced biologic therapies for chronic inflammatory conditions, has resulted in a multiplication in leishmaniasis.

\section{Symptoms/Pathology}

These are mature Infection with Leishmania species can result in 3 types of disease depending on the species, geographic region and host immune response.

1. Leishmania donovani produces visceral leishmaniasis (kala-azar): Symptoms include fever (often 2 fever spikes per day), expansion of the spleen and liver, weakness, and continuing emaciation. The disease is often fatal without treatment, but survivors often develop immunity.

2. Leishmania tropica and L. mexicana produce cutaneous leishmaniasis: which is distinguished by skin lesions (oriental sore). Infected macrophages having amastigotes are found predominantly at the site of infection around the sores. The sores are characterized by a rise rim encircling the lesion. The sores generally heal by themselves within a year, but secondary bacterial infections are potential in open sores [1-5].

3. Leishmania braziliensis produces mucocutaneous leishmaniasis: characterized by lesions near mucosal membranes. The beginning site if infection is a small red papule that ulcerates in a few weeks. The lesions are flat (no raised rim) and often oozing. Infections of the ear, nose and mouth area lead to degeneration of the cartilage and soft tissues, resulting in disfigurement.

\section{Life Cycle}

Diff steps:-

a. While taking a blood meal, the sand- fly liberate promastigotes through the proboscis into the skin.

b. 2. Macrophages phagocytize the promastigotes.

c. Promastigotes convert into amastigotes. 
d. Amastigotes proliferate in cells and macrophages and throughout this time, the signs and symptoms of the disease become particularly prevalent.

e. The sand fly takes a blood meal and ingests macrophages having amastigotes. f. Amastigotes reach the infective stage when they convert into promastigotes in the sandfly's midgut.

g. Promastigotes transfer to the proboscis, ready to be released during the next blood meal (Figure 1).

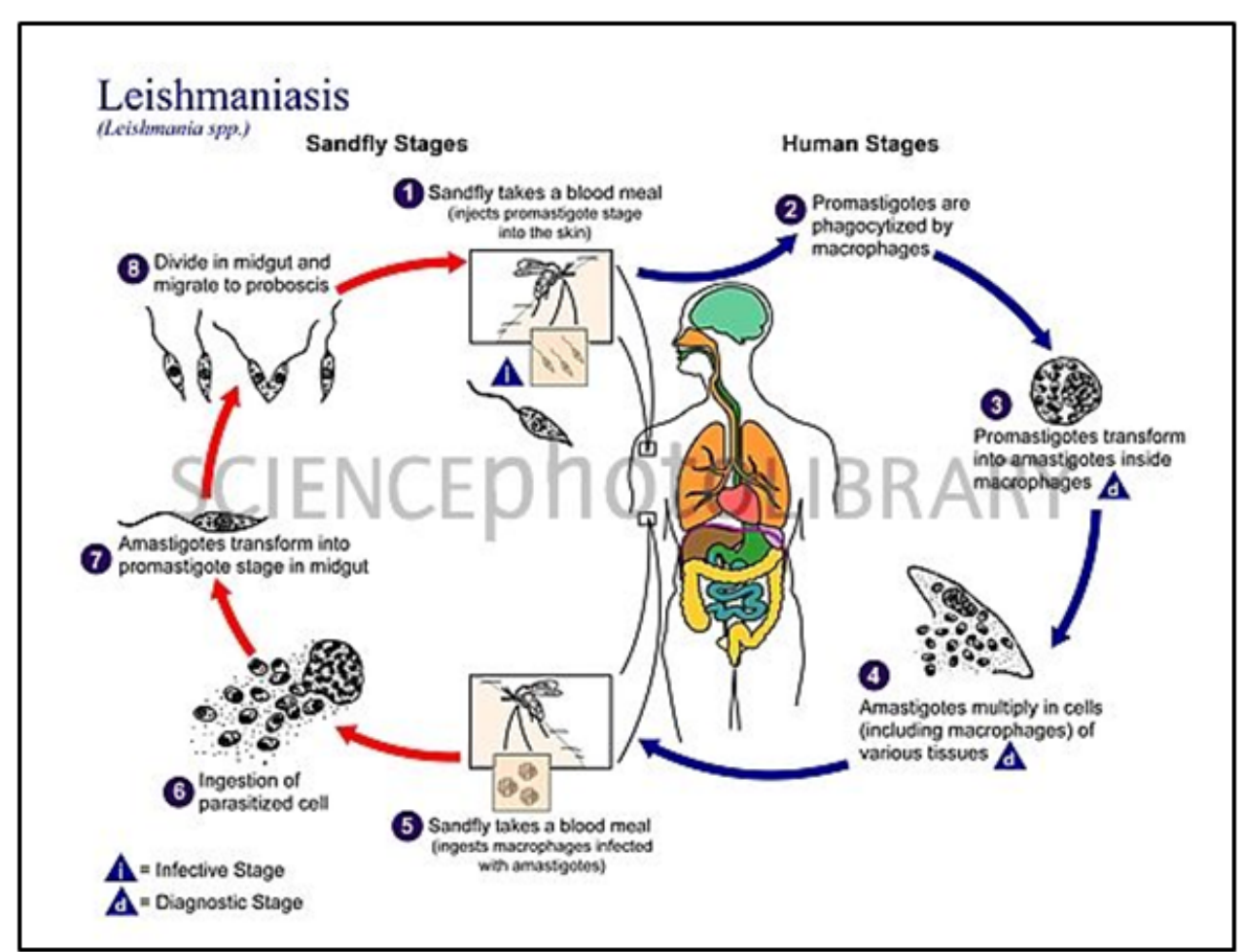

Figure 1 : Life cycle.

\section{Diagnose}

\section{Diagnosing Cutaneous Leishmaniasis}

Your doctor may take a little amount of skin for a biopsy by sweeping one of the ulcers. They'll study the samples under a microscope or in a culture to distinguish the parasite. A culture is a way to see if there are parasites in a sample. It gives a small amount of parasites the opportunity to grow to detectable levels.

\section{Diagnosing Visceral Leishmaniasis}

Many times, people don't recall a bite from a sand fly or a skin sore. This condition may be difficult to diagnose. A doctor may first perform a physical exam to look for an explanation spleen or liver. They may then perform a bone marrow biopsy or take a blood sample for examination. They'll study these samples for the parasite. Diagnosis may take two to four weeks if a culture is necessary.

\section{Treatment}

In addition to be Anti parasitic drugs, such as amphotericin B, treat this condition.

\section{Cutaneous Leishmaniasis}

Cutaneous ulcers will frequently heal without treatment. However, treatment can speed healing and decrease scarring. It can stop the development of further disease. Ulcers on the face that cause damaget may require plastic surgery.

\section{Visceral Leishmaniasis}

Visceral disease needs treatment. Some medications are available. The main types of medicine used are compounds that contain antimony. These include meglumine antimoniate and sodium stibogluconate.

\section{Mucocutaneous Leishmaniasis}

These lesions don't heal naturally. They require some treatment.

Liposomal amphotericin B and paromomycin can treat mucocutaneous leishmaniasis. WHO launched an advocacy campaign to help reduce the price of these drugs. The program reduced the price of liposomal amphotericin B by 90 percent and meglumine antimoniate by 60 percent. The hope is that lowering the cost will make it easier for people to get these treatments.

\section{References}

1. P Desjeux (2004) Leishmaniasis: current situation and new perspectives. Comparative Immunology, Microbiology \& Infectious Diseases 27(5): 305-3185.

2. Philippe Minodier, Philippe Parola (2007) Cutaneous leishmaniasis treatment. Travel Medicine and Infectious Disease 5(3): 150-158. 
3. David Pace (2014) Leishmaniasis. Journal of Infection p 1-9.

4. Field V, Gautret P, Schlagenhauf P, Burchard GD, Caumes E, et al. (2010) Travel and migration associated infectious diseases morbidity" in Europe, 2008. BMC Infect Journal of Hepatology 58: 625-629.
5. Murray HW (2005) Advances in leishmaniasis. Lancet 366(9496): 1561-1577.

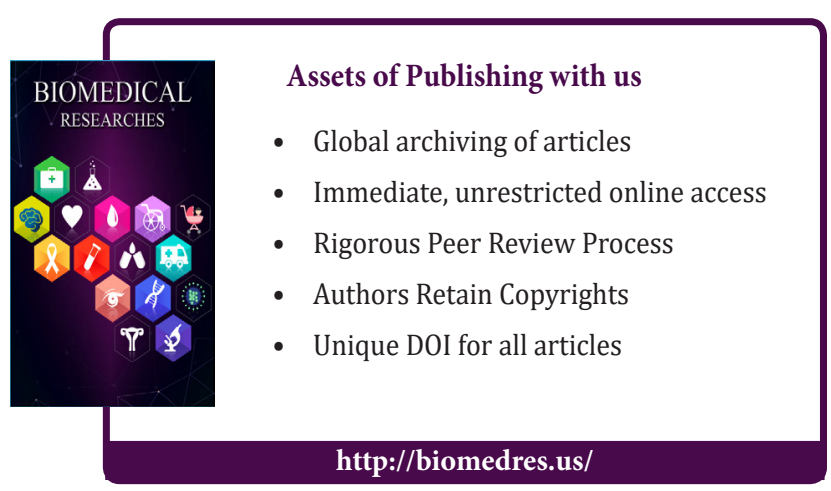

